\title{
A Compact Microwave Applicator for the Rapid Detection of Clostridium Difficile
}

\author{
H. Hamzah ${ }^{1,3}$, E. Ahortor ${ }^{2}$, D. Malyshev ${ }^{2}$, H. Choi ${ }^{1}$, J. Lees ${ }^{1}$, L. Baillie ${ }^{2}$ and A. Porch ${ }^{2}$ \\ ${ }^{1}$ Centre for High Frequency Engineering, School of Engineering, Cardiff University, Cardiff CF24 3AA, U.K. \\ ${ }^{2}$ School of Pharmacy and Pharmaceutical Sciences, Cardiff University, Cardiff, CF10 3NB, U.K. \\ ${ }^{3}$ College of Engineering, University of Al-Qadisiyah, Diwaniyah, Iraq
}

\begin{abstract}
Clostridium difficile is a major cause of hospital acquired infection that poses significant diagnostic challenges. In this paper, a new type of resonant microwave applicator is proposed for the liberation of DNA from $C$. difficile spores via microwave disruption, followed by rapid detection using a sandwich hybridization assay. A split-ring resonator is designed to operate at $2.4 \mathrm{GHz}$ with a $3 \mathrm{~mm}$ active gap region that contains an isolate volume of approximately $10 \mathrm{~mm}^{3}$, exposed to pulsed microwaves of $12 \mathrm{~W}$ rms power. The parallel electric field configuration maximizes the interaction between the microwaves and the sample. In a proof of principle study, in combination with pathogen specific DNA probes, we have used the system to correctly identify virulent strains of $C$. difficile using magnetic bead extraction of DNA suitable for point-of-care application.
\end{abstract}

\section{INTRODUCTION}

In this paper we describe a method for the release of DNA from Clostridium difficile ( $C$. difficile), although the approach is extendable to the detection of other types of bacteria. $C$. difficile is a spore-forming, toxin-producing bacterium which is currently the principal cause of healthcare-associated diarrhoea in the developed world. Specifically, there were 285,000 cases of $C$. difficile infection in the USA in 2007 while the pathogen was linked to the death of over 8,000 individuals in the UK over the same period. Thus, the bacterium presents a major challenge to healthcare professionals and has stimulated development of improved diagnostics and medical countermeasures [1] to help combat $C$. difficile infection rates of almost 500000 pa in the USA [2], over 14000 pa in the UK [3].

The rapid and accurate identification of $C$. difficile in clinical samples, such as faeces, is a key step in reducing the devastating impact of this disease. The current "gold standard" for $C$. difficile diagnosis is the cell cytotoxin neutralization assay (CCTA), which takes between 48-72 hours to generate a result [4],[5]. Faster detection methods have been developed, such as enzyme immunoassays (EIAs) which target the pathogen's two toxins A and B, and glutamate dehydrogenase [5],[6]. These assays are easy to perform and give rapid results (in 15-20 minutes) but suffer from low sensitivity [7]-[10]. Nucleic acid amplification tests (NAATs) are more sensitive than immunoassays, but are expensive and labor intensive. The most rapid (Cepheid Xpert) still takes around 2 hours to provide a result due to the need to include a purification step to remove the biological materials which inhibit signal amplification [11]-[13].
To address the issue of timely diagnosis, a DNA-based detection system for human faeces has been developed [1], where DNA released as a consequence of microwave mediated cellular disruption is detected by pathogen specific DNA probes in less than 20 mins. A pair of triangular gold electrodes were used to "focus" the microwave electric field of a domestic, magnetron-based microwave oven at $2.45 \mathrm{GHz}$. Although effective for rapid DNA release, there are repeatability issues due to the uncertainties related to size of the local electric field. It is also difficult to control the microwave power delivered to the sample, since neither the magnetron frequency nor output power can be adjusted during a single experiment. The use of a magnetron also demands a mains power supply, with safety issues associated with high microwave power levels $(1 \mathrm{~kW} \mathrm{rms})$ and high DC voltages $(5 \mathrm{kV})$ for the magnetron's operation. Hence, magnetron-based methods are clearly unsuitable for incorporation into portable, hand-held diagnostics.

In this work, we have opted to use a solid state source and microwave power amplifier for microwave generation, coupled to a compact, highly efficient applicator (a split ring resonator, SRR). Whilst the system still operates at around $2.45 \mathrm{GHz}$, its compact size means that much lower microwave power levels are needed to achieve similar electric field levels as those of a microwave oven, and in the flow system we have developed only $12 \mathrm{~W}$ rms of microwave power is needed for effective target DNA release. The use of solid state high-frequency electronics means that the precise excitation frequency can be varied during the course of an experiment, to ensure optimal microwave power transfer to the sample. Finally, the design of the resonator permits maximum coupling of the microwave electric field to the sample by ensuring that the sample capillary is oriented parallel to the electric (E) field within the very well-defined E-field antinode of the SRR.

\section{EXPERIMENTAL DETAILS}

We have previously demonstrated the effectiveness of a similar, parallel E-field orientation SRR for liquid sensing [14]. The maximizing of the electric dipole moment for sensing is the same optimization process as ensuring the maximum Efield amplitude for rapid DNA release. A schematic diagram of the copper SRR is shown in Fig. 1, and a COMSOL Multiphysics simulation of its E-field is shown in Fig.2. 

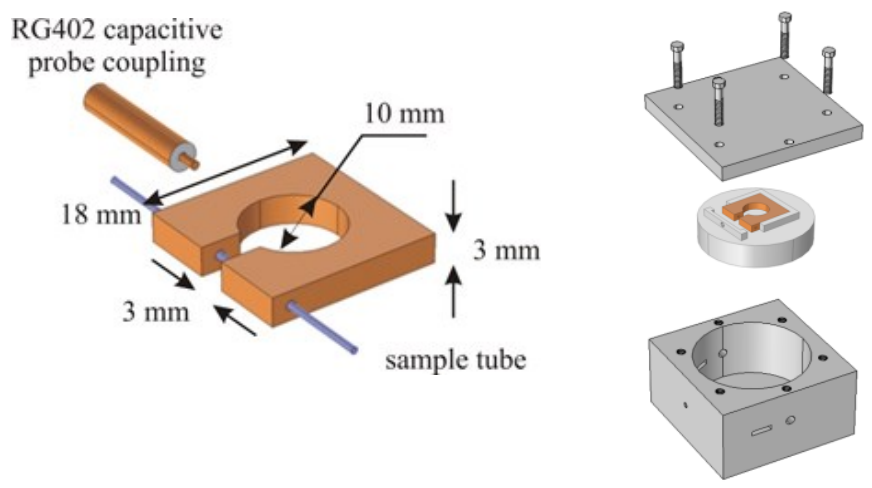

Figure 1. Schematic diagram of the SRR showing the sample location, with a single, adjustable capacitive coupling port (probe terminated RG402 cable). Also shown (on the right) is the full SRR assembly, with the SRR mounted on a PTFE platform and enclosed in an aluminum package for high $Q$.

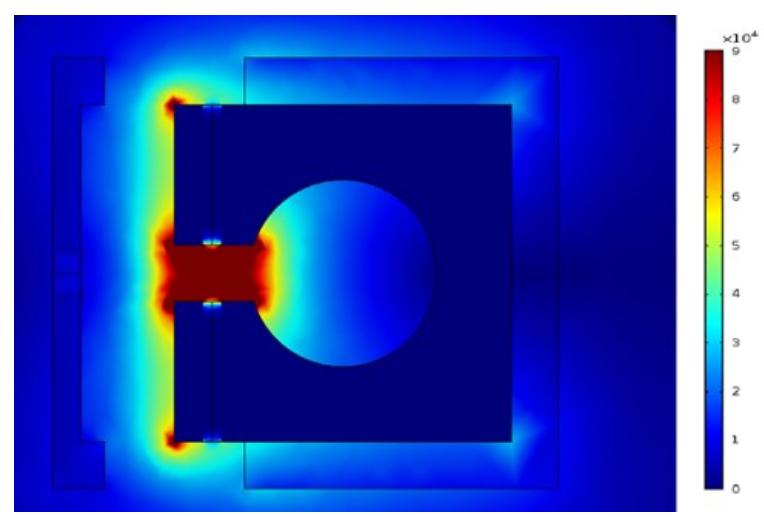

Figure 2. COMSOL Multiphysics simulation showing the E-field amplitude distribution of the SRR it its fundamental (lumped element) mode at $2.4 \mathrm{GHz}$ for an input power of $1 \mathrm{~W}$ rms, illustrating the high field region in the gap.

Referring to Fig. 1, the SRR is located on a low-loss PTFE dielectric platform, which has negligible effect on its quality factor $(Q)$. This assembly is located inside a rectangular aluminum outer cavity, to reduce radiation losses and to maintain the highest possible $Q$, which is around 2000 when the sample is absent. The sample tube and coupling probe enter the structure through a small slot and a feedline hole, respectively. Following adjustment, the feed lines are secured with the aid of two small grub screws. The sample tube is either a quartz capillary of inner and outer diameters of $0.7 \mathrm{~mm}$ and $1.0 \mathrm{~mm}$, respectively, or for flowing samples an FEP (Fluorinated Ethylene Propylene) tube, of outer diameter of $0.77 \mathrm{~mm}$ and inner diameter of $0.40 \mathrm{~mm}$, which can be then connected to a precision flow pump.

The detailed microwave delivery system is shown in Fig. 3. The microwave (MW) synthesizer generates a signal which is passed through the RF switch and directional coupler arrangement. Switching between high $(10 \mathrm{~mW})$ and low $(0.1$ $\mathrm{mW}$ ) power states allows the duty cycle of the microwave exposure to be adjusted. The power amplifier has a gain of $50 \mathrm{~dB}$. The variable attenuator enables fine tuning of the power delivery to the SRR up to $20 \mathrm{~W}$ rms. Fine power adjustment is provided via a LabVIEW software interface to the MW synthesizer itself. The microwave circulator protects the power amplifier from power reflected by the SRR when it is offresonance. Two USB power sensors measure the incident and reflected power to/from the SRR, which allow the power delivery efficiency and actual power delivered into the isolate (which forms the sample) to be calculated.

Whilst we can ensure that maximum power is transferred from microwave source to cavity by critical coupling (i.e. an insertion loss at resonance of at least $-20 \mathrm{~dB}$ ), it is also important that the greatest portion of power delivered to the resonator is absorbed by the sample and not by the resonator itself. Defining $Q_{\mathrm{s}}$ to be the quality factor of the resonator with the sample present and $Q_{0}$ to be that when empty, the quantity $1-Q_{\mathrm{s}} / Q_{0}$ is the fraction of power delivered to the sample. By designing the split ring resonator such that $Q_{\mathrm{s}}<<Q_{0}$, we ensure that almost all of the power delivered is absorbed by the sample. A major factor in achieving this is the parallel sample geometry relative to the applied E field. Typically, $Q_{\mathrm{s}} \approx 200$ and $Q_{0} \approx 2000$, so approximately $90 \%$ of the power delivered into the cavity is absorbed by the sample.

Defining $P_{\text {in }}$ to be the power delivered to the SRR, from the fundamental definition of $Q$ factor we may calculate the mean value of electric field amplitude within the sample using

$$
E_{\mathrm{in}}^{2} \approx \frac{P_{\mathrm{in}}}{\pi f_{0} \varepsilon_{2} \varepsilon_{0} V_{\mathrm{s}}}\left(1-\frac{Q_{\mathrm{s}}}{Q_{0}}\right)
$$

In Eqn.(1), $V_{\mathrm{s}}$ is the sample volume within the gap region, $f_{0}$ is the resonant frequency, $Q_{0}$ and $Q_{\mathrm{s}}$ are the quality factors for the empty and sample-filled SRRs, respectively, and $\varepsilon_{2}$ is the lossy part of the sample's complex relative permittivity (approximately 10 at $2.4 \mathrm{GHz}$, since it is mostly water). For an input power of $12 \mathrm{~W}$ rms, Eqn.(1) predicts an internal electric field $E_{\text {in }} \approx 2 \mathrm{kV} / \mathrm{cm}$. For any other input power $P_{\text {in, }}$, this result can be scaled using a $\sqrt{ } P_{\text {in }}$ dependence.

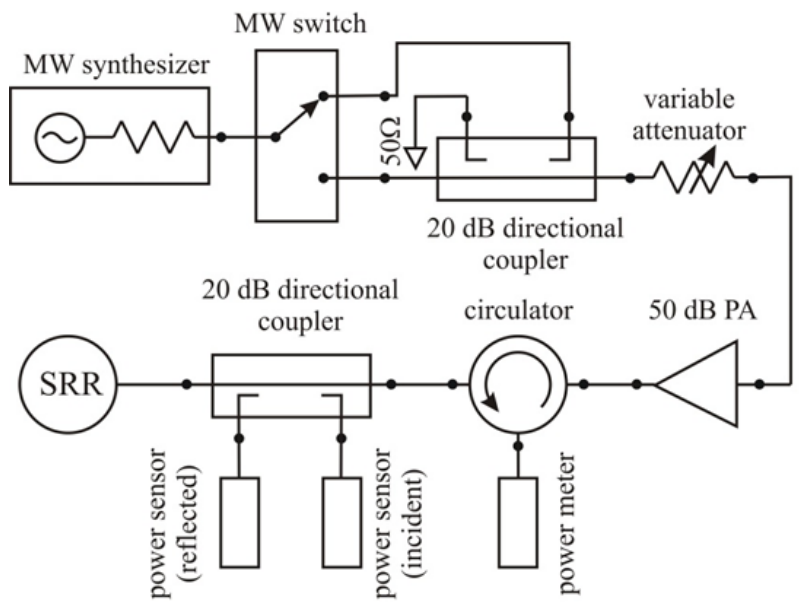

Figure 3. Schematic diagram of the microwave power delivery system. 


\section{EXPERIMENTAL RESULTS AND DISCUSSION}

Microwaves were applied to the isolates with the resonator coaxial feed adjusted for critical coupling at an initial isolate temperature of $25^{\circ} \mathrm{C}$. The initial resonant frequency was observed to be $2.413 \mathrm{GHz}$ in all cases, i.e. at a room temperature of $25^{\circ} \mathrm{C}$. By measuring the reduction in power delivery efficiency during microwave exposure, the increase in temperature of the sample can be assessed. This is very important as heating above $63^{\circ} \mathrm{C}$ causes the DNA to denature. Both real and imaginary parts of the permittivity of water decrease with increasing temperature, increasing both resonant frequency and $Q$, which cause the power delivery efficiency to decrease. Simple modelling using the Debye equations [15] allows us to estimate the sample's temperature increase $\Delta T$ after microwave exposure, which was kept below $25^{\circ} \mathrm{C}$ (i.e. a maximum sample temperature of $50^{\circ} \mathrm{C}$ ) for all experiments.

We studied DNA release from three clinical isolates of $C$. difficile; DS1813 and R20291, which are members of the hypervirulent 027 Ribotype group, and DS1684, which is nonvirulent due to the absence of a gene which encodes a major virulence factor (tcdA). The appearance of the spore form of DS1813 and R20291 differ markedly, allowing us to assess the impact of physical structure on microwave mediated DNA release, as shown in Fig. 4. Purified spores of each isolate were prepared in a category 2 laboratory according to standard methods [16] and were then diluted to a concentration of $10^{5}$ spores $/ \mu \mathrm{l}$ in deionized water (Milli-Q, $18 \mathrm{M} \Omega \mathrm{cm}$ resistivity). The amount of single stranded (ss) DNA released during microwave exposure was quantified using a Qubit 3.0 fluorometer and Qubit ssDNA assay reagent, with $20 \mu \mathrm{l}$ of either microwave treated or untreated $C$. difficile spore suspensions collected via the syringe pump, programmed to maintain a constant isolate flow rate. All ssDNA extraction data were taken in triplicate $(N=3)$ for each microwave exposure and the standard deviations were calculated.

The ssDNA released following microwave treatment was screened for the presence of a tcdA gene target sequence using DNA probes. The nucleotide sequences and chemical modifications of the probes are shown in Table 1 and were synthesized and purchased from Invitrogen (UK) and Biomers (Germany), respectively. The most effective microwave protocol was found to be at $40 \%$ duty cycle, with $1000 \mathrm{~ms}$ pulse period, $400 \mathrm{~ms}$ ON time, at $12 \mathrm{~W}$ rms power and constant sample flow rate of $400 \mu \mathrm{l} / \mathrm{h}$.

TABLE I

SEQUENCES OF THE DNA PROBES USED IN THIS STUDY

\begin{tabular}{|l|l|}
\hline Probe Name & Sequences (5'-3') \\
\hline tcdA anchor probe & Biotin-TTTTTTTTAATACTAACACTGC \\
\hline tcdA reporter probe & $\begin{array}{l}\text { TGTTGCAGTTACTGGATGGCAA-HRP } \\
\text { HRP }=\text { horseradish peroxidase }\end{array}$ \\
\hline
\end{tabular}

(a)

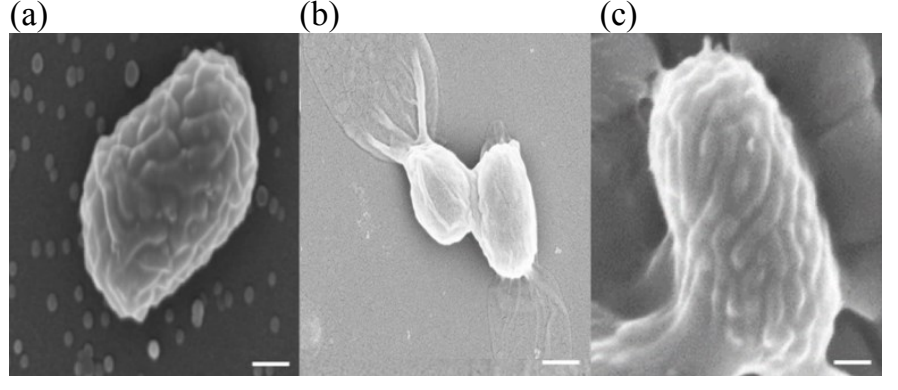

Figure 4. Scanning electron microscopy images of structurally distinct $C$. difficile spores: (a) DS1684 (b) R20291; (c) DS1813. Each spore was magnified by a factor of 30,000 . The scale bar corresponds to $200 \mathrm{~nm}$.

Given that our over-arching aim is to develop a system capable of detecting $C$. difficile specific DNA targets, we also determined the feasibility of using activated magnetic particles linked to a DNA probe specific for a major virulence factor (tcdA), to capture ssDNA after microwave exposure. These particles can be easily collected using controllable magnetic fields following microwave exposure. The MagRack6 magnetic bead separation system (by Sigma-Aldrich) was used, followed by colorimetric detection of $C$. difficile ssDNA using a sandwich hybridization assay (SHA).

The principle of SHA is the hybridization of the target gene between anchor (5' biotin modified) and reporter (3' HRP modified) probes, to form a 3-strand DNA complex. The complex is then trapped and identified by the addition of the streptavidin coated magnetic beads. In a typical SHA, $5 \mu \mathrm{L}$ of magnetic bead captured biotin probe was incubated with microwaved bacterial suspension $(20 \mu \mathrm{L})$, HRP reporter probe $(5 \mu \mathrm{L}, 1 \mu \mathrm{M})$ and $20 \mu \mathrm{L}$ of hybridization buffer (PBS- $0.1 \%$ Tween 20). The mixture was incubated at $50^{\circ} \mathrm{C}$ in an orbital shaker at $200 \mathrm{rpm}$ for 60 minutes. Hybridized 3-strand DNA complexes were captured by an applied magnetic field and washed three times with hybridization buffer. The presence of the target DNA was determined by the addition of $100 \mu \mathrm{L}$ of TMB solution, a substrate for horseradish peroxidase (HRP) which produces a blue color. This is followed by the addition $1 \mathrm{M} \mathrm{H}_{2} \mathrm{SO}_{4}(100 \mu \mathrm{L})$. The final reaction solution is a yellow coloration with maximal absorbance at $450 \mathrm{~nm}$.

As can be seen from the absorbance data at a wavelength of $450 \mathrm{~nm}$ shown in Fig. 5, we obtained a positive signal for R20291 and DS1813 but not for DS1684, included in this study as a negative control. A no template control (NTC) was also included in the assay. The horizontal line drawn at an absorbance of 0.16 is the cut-off value for a positive test, defined as the mean optical density plus the $3 \sigma$ value of the blank. From the expected positive signal for R20291 and DS1813, but not for DS1684 (which lacked the tcdA gene target), we conclude that the assay has worked successfully. 


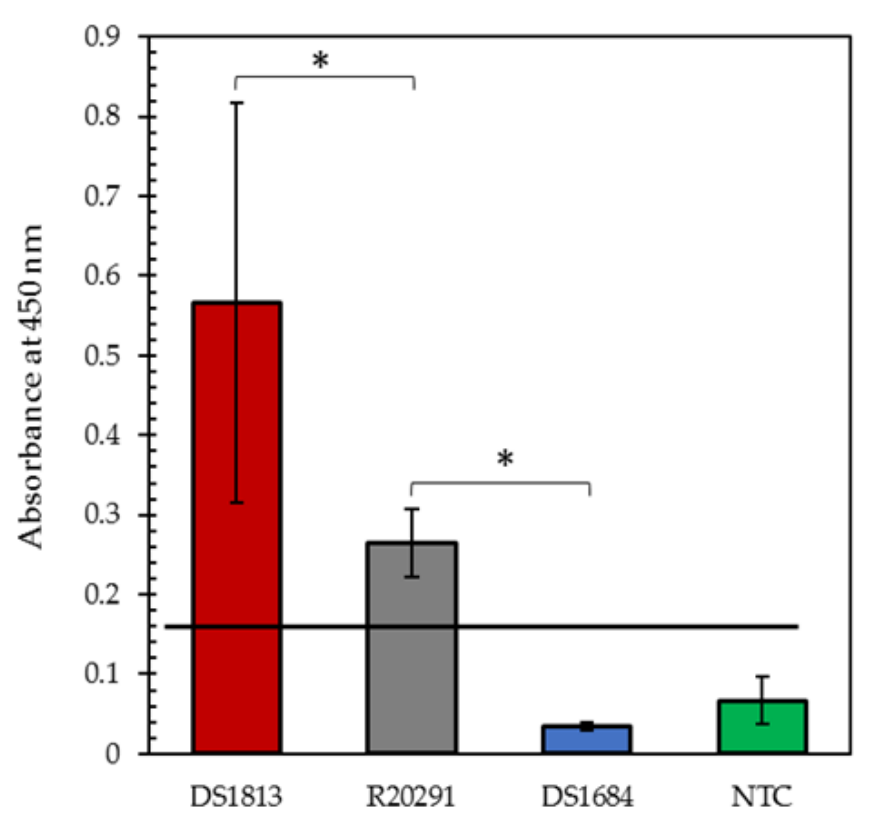

Figure 5. Colorimetric detection of $C$. difficile ssDNA in a sandwich hybridization assay. ssDNA released from spore suspension after microwave excitation at $40 \%$ duty cycle and $12 \mathrm{~W}$ rms power was tested. The data for each strain is the mean value of three replicate experiments with its standard deviation. The cut-off value (horizontal line) for a positive test was defined as the mean optical density plus the $3 \sigma$ value of the blank. Each asterisk represents the statistical significance between groups $(p<0.05)$.

\section{CONCLUSIONS}

A novel, high $Q$, split ring resonator has been proposed as a microwave microfluidic applicator for the rapid release of single stranded DNA from $C$. difficile spores. Its low volume over which the electric energy is concentrated (effectively the gap region, only about $10 \mathrm{~mm}^{3}$ ) and the low amount of electric field depolarization within the sample when it is in the parallel geometry provide a dual role as an effective sensor and microwave power applicator; the second function has been investigated in detail in this paper. The most effective microwave protocol for spontaneous liberation of single stranded DNA was found to be at $40 \%$ duty cycle with $12 \mathrm{~W}$ rms power, measured using a Qubit 3.0 fluorometer and Qubit ssDNA assay reagent. Towards the development of a hand held diagnostic device, we have also demonstrated an effective magnetic particle ssDNA extraction method. With this in mind, we next aim to reduce the microwave power level down to around $1 \mathrm{~W}$ rms based on further optimization of the microwave exposure protocol and the sample flow rate through the split ring resonator's gap region. In parallel, we will also optimize the sandwich hybridization assay to reduce the time taken for this step from 60 mins to around 20 mins.

\section{REFERENCES}

[1] L.T. Joshi, B.L. Mali, C.D. Geddes and L. Baillie, "Extraction and sensitive detection of toxins $\mathrm{A}$ and $\mathrm{B}$ from the human pathogen Clostridium difficile in 40 seconds using microwave-accelerated metalenhanced fluorescence", PLoS One, vol. 9, e104334. Aug 2014.

[2] CDC, 2015 Health Security Report, U.S. Department of Health and Human Services Centers for Disease Control and Prevention, 2015.

[3] https://www.gov.uk/government/statistics/clostridium-difficile-infectionannual-data. Clostridium difficile infection: annual data. 2016

[4] S.M. Poutanen and A.E. Simor, "Clostridium difficile-associated diarrhea in adults", Canadian Medical Association Journal, vol. 171, pp. 51-58, Jul 2004.

[5] S.D. Goldenberg, P.R. Cliff, S. Smith, M. Milner and G.L. French, "Two-step glutamate dehydrogenase antigen real-time polymerase chain reaction assay for detection of toxigenic Clostridium difficile", J. Hosp. Infect., vol. 74, pp. 48-54, Jan 2010.

[6] A. Arnold et al., "Prospective assessment of two-stage testing for Clostridium difficile", J. Hosp. Infect., vol. 76, pp. 18-22, Sep 2010.

[7] S.D. Bélanger, M. Boissinot, N. Clairoux, F.J. Picard and M.G. Bergeron, "Rapid Detection of Clostridium difficile in feces by real-time PCR", Journal of Clinical Microbiology, vol. 41, pp. 730-734, Feb 2003.

[8] T. Planche et al., "Diagnosis of Clostridium difficile infection by toxin detection kits: a systematic review", Lancet Infect. Dis., vol. 8, pp. 777784, Dec 2008.

[9] F.C. Tenover et al., "Impact of strain type on detection of toxigenic Clostridium difficile: comparison of molecular diagnostic and enzyme immunoassay approaches". J. Clin. Microbiol., vol. 48, pp. 3719-3724, Oct 2010.

[10] R.J. Carman et al., "Glutamate dehydrogenase is highly conserved among Clostridium difficile ribotypes”, J. Clin. Microbiol., vol. 50, pp. 1425-1426, Apr 2012.

[11] C.W. Knetsch et al., "Comparison of real-time PCR techniques to cytotoxigenic culture methods for diagnosing Clostridium difficile infection”. J. Clin. Microbiol., vol. 49, pp. 227-231, Jan 2011.

[12] T. Rinttilä, A. Kassinen, E. Malinen, L. Krogius and A. Palva, "Development of an extensive set of 16S rDNA-targeted primers for quantification of pathogenic and indigenous bacteria in faecal samples by real-time PCR", J. Appl. Microbiol., vol. 97, pp. 1166-1177, Jun 2004.

[13] K. Eastwood, P. Else, A. Charlett and M. Wilcox, "Comparison of nine commercially available Clostridium difficile toxin detection assays, a real-time PCR assay for $C$. difficile tcdB, and a glutamate dehydrogenase detection assay to cytotoxin testing and cytotoxigenic culture methods", $J$. Clin. Microbiol., vol. 47, pp. 3211-3217, Oct 2009.

[14] H. Hamzah,, J. Lees and A. Porch, "Split ring resonator with optimized sensitivity for microfluidic sensing", Sensors and Actuators A: Physical, vol. 276, pp.1-10, Jun 2018

[15] U. Kaatze, "Complex permittivity of water as a function of frequency and temperature". J. Chem. Eng. Data, vol. 34, pp. 371-374, Oct 1989.

[16] J.A. Sorg, and A.L. Sonenshein, "Bile salts and glycine as cogerminants for Clostridium difficile spores", J. Bacteriol., vol. 190, pp. 2505-2512, Apr 2008. 